\title{
Recent progress in brightness scaling by coherent beam combining of tapered amplifiers for efficient high power frequency doubling
}

Albrodt , P. ; Jamal, Muhammad Tahir; Hansen, Anders Kragh; Jensen, Ole Bjarlin; Niemeyer, M. ; Blume, G. ; Paschke, K.; Crump, P.; Hamperl, J.; Georges, P.

Total number of authors:

11

Published in:

Proceedings of SPIE

Link to article, DOI:

$10.1117 / 12.2508443$

Publication date:

2019

Document Version

Peer reviewed version

Link back to DTU Orbit

Citation (APA):

Albrodt , P., Jamal, M. T., Hansen, A. K., Jensen, O. B., Niemeyer, M., Blume, G., Paschke, K., Crump, P., Hamperl, J., Georges, P., \& Lucas-Leclin, G. (2019). Recent progress in brightness scaling by coherent beam combining of tapered amplifiers for efficient high power frequency doubling. In Proceedings of SPIE (Vol. 10900). [1090000] SPIE - International Society for Optical Engineering. Proceedings of SPIE - The International Society for Optical Engineering https://doi.org/10.1117/12.2508443

\section{General rights}

Copyright and moral rights for the publications made accessible in the public portal are retained by the authors and/or other copyright owners and it is a condition of accessing publications that users recognise and abide by the legal requirements associated with these rights.

- Users may download and print one copy of any publication from the public portal for the purpose of private study or research.

- You may not further distribute the material or use it for any profit-making activity or commercial gain

- You may freely distribute the URL identifying the publication in the public portal 
See discussions, stats, and author profiles for this publication at: https://www.researchgate.net/publication/331596422

Recent progress in brightness scaling by coherent beam combining of tapered amplifiers for efficient high power frequency doubling

Conference Paper $\cdot$ March 2019

DOI: $10.1117 / 12.2508443$

\section{CITATIONS}

0

11 authors, including:

\section{Philipp Albrodt}

Institut d'Optique Graduate School

8 PUBLICATIONS 5 CITATIONS

SEE PROFILE

Ole Bjarlin Jensen

Technical University of Denmark

102 PUBLICATIONS 698 CITATIONS

SEE PROFILE
READS

27

Muhammad Tahir Jamal

Technical University of Denmark

5 PUBLICATIONS 1 CITATION

SEE PROFILE

Gunnar Blume

Ferdinand-Braun-Institut

90 PUBLICATIONS 589 CITATIONS

SEE PROFILE

Some of the authors of this publication are also working on these related projects:

Project Laser stacs for nuclear fusion View project

Project borates View project 


\title{
Recent progress in brightness scaling by coherent beam combining of tapered amplifiers for efficient high power frequency doubling
}

\author{
P. Albrodt ${ }^{\text {a }}$, M. T. Jamal ${ }^{\text {b }}$, A. K. Hansen ${ }^{\text {b }}$, O. B. Jensen ${ }^{\text {b }}$, M. Niemeyer ${ }^{\text {c }}$, G. Blume $^{\text {c }}$, K. Paschke $^{\text {c }}$, \\ P. Crump ${ }^{c}$, J. Hamperl ${ }^{\text {a }}$, P. Georges ${ }^{\mathrm{a}}$ and G. Lucas-Leclin*a \\ ${ }^{a}$ Laboratoire Charles Fabry, Institut d'Optique Graduate School, CNRS, Université Paris Saclay, \\ 91127 Palaiseau Cedex, France \\ ${ }^{b}$ DTU Fotonik, Department of Photonics Engineering, Technical University of Denmark, \\ Frederiksborgvej 399, 4000 Roskilde, Denmark \\ ${ }^{c}$ Ferdinand-Braun-Institut, Leibniz-Institut für Höchstfrequenztechnik, Gustav-Kirchhoff-Str. 4, \\ 12489 Berlin, Germany
}

*gaelle.lucas-leclin@institutoptique.fr

\begin{abstract}
High brightness diode laser beam combining techniques are in demand for efficient high power nonlinear conversion. Coherent beam combining (CBC) is the only method that has the potential for brightness scaling by maintaining one single narrow spectral linewidth. CBC in a master oscillator power amplifier (MOPA) configuration using a small number of efficiently cooled tapered amplifiers is a promising approach for efficient brightness scaling in a simple architecture. We present the application of such a source based on CBC of three tapered amplifiers seeded by a DFB laser at $\lambda=976 \mathrm{~nm}$ for second harmonic generation (SHG). A maximum power of $2.1 \mathrm{~W}$ at $488 \mathrm{~nm}$ was generated by SHG in a MgO:PPLN bulk crystal limited by thermal effects. A clear benefit of the beam clean-up resulting from the CBC setup was documented leading to an improved nonlinear efficiency. As part of our ongoing studies into further brightness scaling in CBC architectures, we present an experimental analysis of the phase dynamics of tapered amplifiers in quasi continuous operation (QCW) at high currents. Furthermore, we are investigating different amplifier designs for improved beam quality at high powers and therefore improved combining efficiency.
\end{abstract}

Keywords: coherent beam combining, tapered laser amplifiers, high-brightness diode lasers, second harmonic generation

\section{INTRODUCTION}

High brightness tapered lasers (TPL) and tapered amplifiers (TPA) combine an excellent beam quality with high powers ${ }^{1}$ and are a promising building block for brightness scaling in beam combining architectures ${ }^{2}$ for example by coherent beam combining (CBC). CBC is the superposition of multiple laser beams by constructive interference. It requires a proper and stable phase relationship of the sampled gain medium. Different approaches have been demonstrated: active phase locking of amplifiers seeded by a single frequency laser split into several beams or passive phase locking of emitters in an extended cavity $^{3}$. Previous studies into CBC of tapered lasers have shown that active phase locking in a master oscillator power amplifier configuration allows to benefit from the full power potential of such devices ${ }^{4,5}$.

TPAs usually have a high power content in the diffraction limited central lobe (60-80\%) while the rest of the optical power is distributed in higher angle side lobes and does not effectively contribute in high brightness applications. CBC of individual TPAs is one way to combine the diffraction limited content of such beams and filter out the stray light in a simple optical setup. Then, the brightness level in the near infrared (NIR) can be scaled efficiently, while a narrow spectral linewidth is maintained.

We demonstrate in this paper the importance of CBC architectures for the development of high power lasers in the visible spectral range based on second harmonic generation (SHG). Efficient SHG requires a good beam quality, a narrow spectral linewidth and a high input power ${ }^{6}$. Diode laser based visible light sources have become increasingly attractive for 
biomedical applications ${ }^{7}$. Among other approaches to power scaling of frequency converted diode lasers ${ }^{8}$, CBC of tapered lasers is one way to improve the power and spatial properties of the NIR pump source and allows to reach higher powers of the converted beam as described later in subsection 2.2.

In order to improve the combining efficiency and to increase the optical power, we are investigating new designs of TPAs. A higher beam quality of the amplifiers directly leads to a higher combining efficiency for CBC at high powers. We report on investigations of different device configurations, leveraging both improved epitaxial structures and tapered amplifier designs to improve the performance of future CBC setups. Furthermore, we demonstrate CBC in pulsed regime as a potential pathway for further power scaling.

\section{BRIGHTNESS SCALING BY CBC}

\subsection{CBC interferometer}

Our CBC-architecture consists of a multiarm-interferometer and three individual TPAs, with active phase control by feedback on the amplifier currents (Fig. 1). A narrow-linewidth single-mode DFB laser diode emitting at $\lambda=976 \mathrm{~nm}$ is used as the seed source and is optically isolated by a double stage Faraday isolator with an isolation higher than $-50 \mathrm{~dB}$. The detailed optical setup of the CBC interferometer is described in reference 5. The design of the amplifiers was taken from reference 9 . We used $6 \mathrm{~mm}$ long amplifiers with $2 \mathrm{~mm}$ ridge waveguide $(\mathrm{RW})$ and $4 \mathrm{~mm}$ tapered section $\left(\mathrm{TP}, 6^{\circ}\right.$ taper angle) mounted on $\mathrm{CuW}$-submounts and C-mounts. This device design is in the following referred as the "reference design". Each amplifier reaches a maximum optical power of $6.5 \mathrm{~W}$ with $4.6 \mathrm{~W}(70 \%)$ contained in the central lobe. The CBC-architecture delivers up to $12.9 \mathrm{~W}$ in $\mathrm{CW}$ at a CBC-efficiency of $>65 \%$, which is limited by the beam quality of the amplifiers ${ }^{5}$.

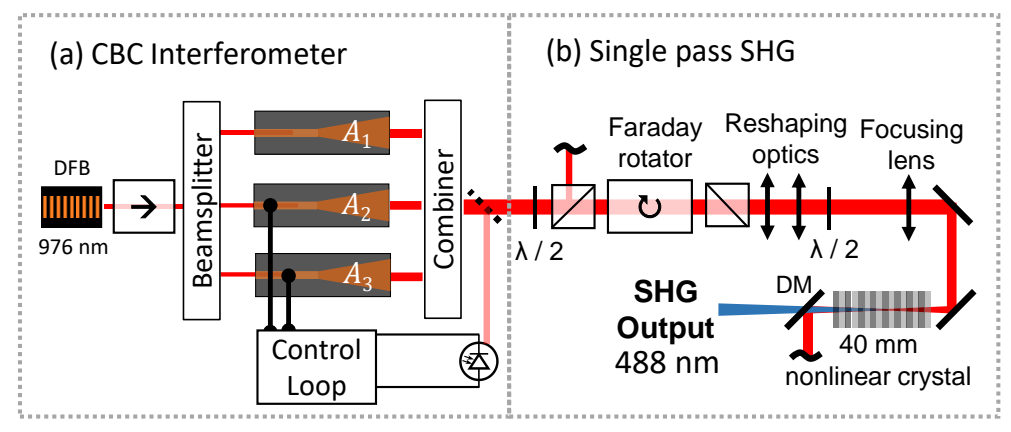

Figure 1 Experimental setup of (a) the CBC interferometer in MOPA configuration and (b) the single pass SHG in a $40 \mathrm{~mm}$ long MgO:PPLN bulk crystal.

One can consider the beam of a tapered laser as a sum of the fundamental mode, corresponding to the central lobe, and emission at high angle ("side lobes"). The central lobes of each beam are very similar and interfere constructively with a high combining efficiency. But the high-order modes exhibit a significantly higher beam mismatch and get therefore partially filtered during the CBC. Furthermore, amplified spontaneous emission is incoherent to the seed source and is therefore weakened by $50 \%$ at each beam combiner. Consequently, CBC of beams from tapered lasers leads to a cleanup of the spatial beam profile where the central lobe is maintained and the high angle, non diffraction-limited, emission is suppressed. The beam profile measurements shown in Fig. 2 illustrate this effect. Those beam profiles correspond to the beam waist after the focusing lens with a $1 / \mathrm{e}^{2}$ waist diameter of about $95 \mu \mathrm{m}$. One can clearly see that the side lobes in slow axis (SA) get filtered by CBC. The power content in the central lobe gets increased from $71 \%$ for one individual amplifier to $86 \%$ for $\mathrm{CBC}$ of $\mathrm{A}_{1+2+3}$. As a result the beam quality factor is reduced from $M_{4 \sigma}^{2}<1.3 \times 4$ for one individual amplifier to $M_{4 \sigma}^{2}<1.1 \times 2.5$ for the final beam. Consequently the experimental combining efficiency of our setup is mostly limited by the power losses induced by this beam clean-up, which removes the undesired high-angle side lobes of the amplifier beams. 


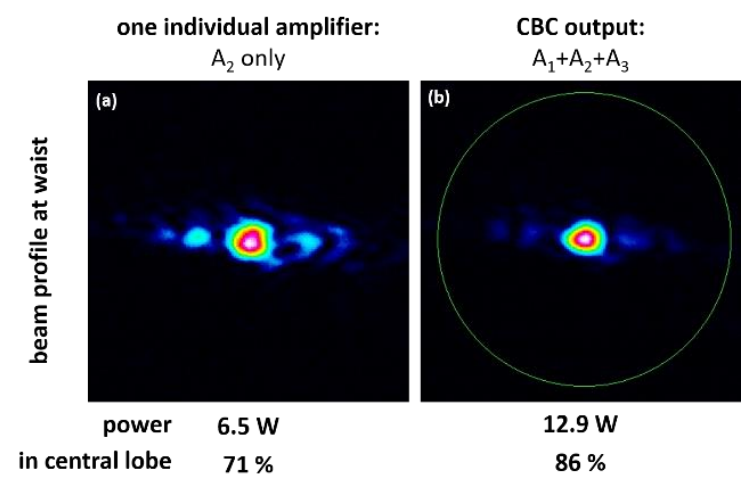

Figure 2. Beam profiles at waist for (a) one individual amplifier (reference design) and (b) the CBC output $\mathrm{A}_{1+2+3}$. Measurement for $\mathrm{I}_{\mathrm{tp}}$ $=10 \mathrm{~A}, \mathrm{~T}=20^{\circ} \mathrm{C}$ and actively controlled ridge currents in the range of 250 to $400 \mathrm{~mA}$. The FA is in vertical and the SA is in horizontal direction. The given central lobe power content was calculated by fitting a Gaussian beam profile to the central lobe of the beam and calculating the power content within the fitted profile ${ }^{10}$.

Measurements of the spectrum showed that the spectral properties of the DFB seed laser were maintained during CBC. The measured spectrum at maximum power is shown in Figure 3. The side mode suppression ratio was better than $40 \mathrm{~dB}$. The full width at half maximum (FWHM) was smaller than $20 \mathrm{pm}$, limited by the resolution of the optical spectrum analyzer (OSA). The DFB seed laser was slightly tunable in the range of $+/-0.5 \mathrm{~nm}$. We conclude that CBC of tapered amplifiers in a simple MOPA architecture is a straightforward method for the development of high brightness pump sources with high spectral purity.

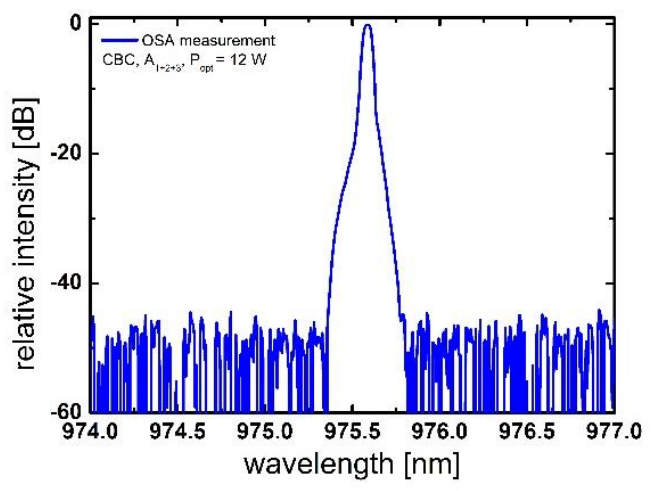

Figure 3. Measured spectrum of the CBC output beam at maximum power. The resolution of the used OSA was $20 \mathrm{pm}$.

\subsection{Single pass second harmonic generation}

The output of the CBC-interferometer was used for single pass SHG as shown in Fig. 1. In order to test the nonlinear conversion efficiency at different levels of brightness, we used the beam from either a single amplifier $\left(\mathrm{A}_{2}\right)$ or from the CBC of three power amplifiers $\left(\mathrm{A}_{1+2+3}\right)$ as the input pump beam for the SHG. During the SHG experiments all three amplifiers were operated at constant current into the tapered section $\left(I_{t p}=9\right.$ A) and the power used for SHG was adjusted by turning the polarization before the first polarizing beam splitter (PBS). The optical isolation between the CBC interferometer and the SHG experiment was $>25 \mathrm{~dB}$. After reshaping optics for beam size adjustment and astigmatism correction, another half-wave plate is used to align the polarization of the NIR beam with the crystallographic Z-axis of the nonlinear crystal which is parallel to the thickness of the crystal in this case. The NIR beam was then focused with a lens into the $40 \mathrm{~mm}$ long periodically poled $\mathrm{MgO}: \mathrm{LiNbO}_{3}(\mathrm{PPLN})$. The $1 / \mathrm{e}^{2}$ waist diameter of the focused beam inside the crystal was measured to be $\sim 95 \mu \mathrm{m}$, which was experimentally verified to be the optimum focusing condition at the highest NIR pump power. The focusing conditions were identical for both pump source configurations. The PPLN crystal was mounted into a temperature-controlled closed-top oven. The temperature was measured with a temperature sensor in the oven, corresponds to an average temperature and was optimized at each pump power level. The spectral components were separated by a dichroic mirror (DM). 
The SHG power was measured for both source configurations: a single amplifier $\left(\mathrm{A}_{2}\right)$ and for CBC of three amplifiers $\left(\mathrm{A}_{1+2+3}\right)$. Results are shown in Fig. 4. We fitted the pump depletion approximation

$$
P_{2 \omega}=P_{\omega} \times \tanh ^{2}\left(\sqrt{\eta P_{\omega}}\right)
$$

where $\eta$ is the nonlinear conversion efficiency, to the experimental values for pump powers $<6 \mathrm{~W}$. The nonlinear conversion efficiencies in case $\mathrm{A}_{2}$ and $\mathrm{A}_{1+2+3}$ obtained by numerical fitting are $2.6 \% / \mathrm{W}$ and $4.5 \% / \mathrm{W}$, respectively. This corresponds to an increase of up to $73 \%$ enhanced by the beam clean-up and the scaled brightness. We attribute the deviation of measured SHG power from the theoretical fit for $\mathrm{P}_{\omega} \geq 6 \mathrm{~W}$ to thermal dephasing due to localized heating caused by SH absorption ${ }^{11}$. This limited the maximum SHG output power to $2.09 \mathrm{~W}$ for $9.2 \mathrm{~W}$ of NIR input power (conversion efficiency $>22 \%$ ). Further increase of the SH power was limited by thermal effects in the PPLN crystal and not by the available pump power or the pump beam quality ${ }^{10}$.

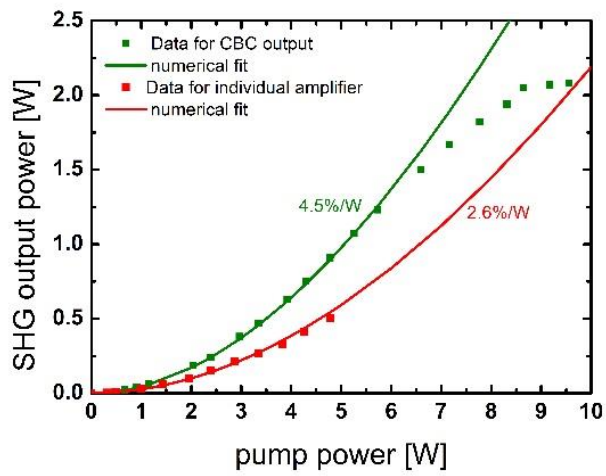

Figure 4. SHG output power $\left(\mathrm{P}_{2 \omega}\right)$ vs. fundamental pump power $\left(\mathrm{P}_{\omega}\right)$ with corresponding numerical fits using pump depletion approximation. The data was taken from reference 10 .

We investigated the long term stability of the SH output beam by power measurements over one hour. The result in Fig. 5(a) shows acceptable stability of the setup with small fluctuations (RMS stability $<0.6 \%$ ) linked to the stability of the CBC interferometer and the critical thermal load in the PPLN. The measured spectral FWHM linewidth of the generated blue-green light $(488 \mathrm{~nm}$ ) was less than $20 \mathrm{pm}$ limited by the resolution of the optical spectrum analyzer. The beam quality of SHG was close to diffraction-limited as shown in Fig. 5(b) with $\boldsymbol{M}_{4 \sigma}^{2}<1.2$ in both directions. The improved beam quality of the SHG beam compared to the fundamental input beam $\left(M_{4 \sigma}^{2}<2.5\right)$ is caused by the so-called nonlinear beam clean-up ${ }^{12}$.

(a)

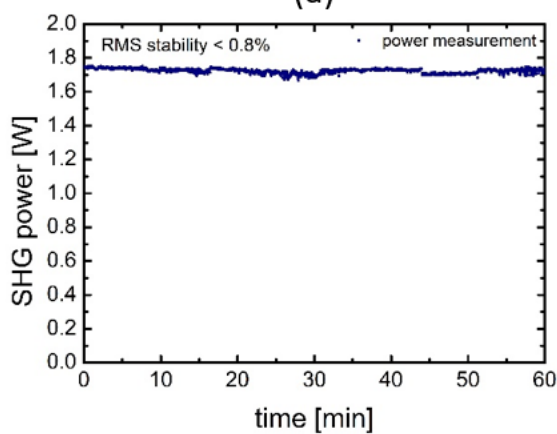

(b)

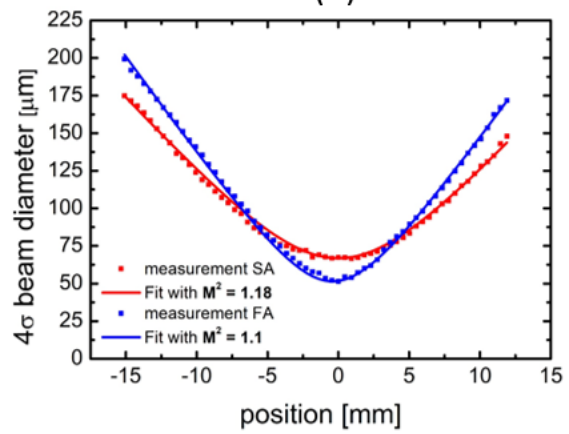

Figure 5. (a) Power stability measurement (one point per second) of the SH beam at $1.7 \mathrm{~W}$ average power and (b) beam quality measurement of the $\mathrm{SH}$ beam at $1.7 \mathrm{~W}$ average power. 


\section{AMPLIFIER DESIGN FOR HIGH-EFFICIENCY CBC}

We want to improve the combining efficiency in our coherent combining setup by advanced design of the amplifier structure. As shown earlier in Fig. 2, the beam-clean up during CBC comes at the cost of power losses as the higher angle side lobes are filtered out. One can expect higher combining efficiencies by making use of tapered amplifiers with an improved power content in the central lobe. The devices described in section 2 (reference design) are in the following compared to the performance of a new amplifier design with an epitaxial structure with an extremely low vertical divergence based on the ELOD2 design ${ }^{13}$. A low vertical divergence is beneficial to the use in a CBC setup, where tolerances in the alignment of the collimation lenses impact the achievable combining efficiency ${ }^{14}$. Furthermore, previous studies have shown that this amplifier design can also deliver high beam quality in slow axis with an improved power content in the central lobe ${ }^{15,16}$. A drawback is, however, its reduced electrical to optical efficiency ${ }^{16}$. We used $5 \mathrm{~mm}$ long devices ( $1 \mathrm{~mm}$ ridge $+4 \mathrm{~mm}$ taper) mounted p-side up on $\mathrm{CuW}$ heat spreaders and $\mathrm{C}$-mounts, with separate electrical contacts in order to control the injection current of ridge $\left(\mathrm{I}_{\mathrm{rw}}\right)$ and tapered section $\left(\mathrm{I}_{\mathrm{tp}}\right)$ independently. The power measurements for the ELOD2 design are shown in Fig. 6(a) in continuous wave (CW) and quasi continuous wave (QCW, $1 \mathrm{~ms}$ on-time, $10 \%$ duty cycle) in comparison to the reference design. We observe a lower CW power for the ELOD2 design with a maximum power of $5 \mathrm{~W}$ at 10 A limited by a significant onset of the thermal rollover at high currents. However, we observe a clear improvement of the power content in the central lobe of the beam. While the reference design has a $>70 \%$ power content at $10 \mathrm{~A}$, we measured $>80 \%$ for the ELOD2 design (Figure $6(\mathrm{~b})$ ), corresponding to $\sim 4 \mathrm{~W}$ in the central lobe.

As the characterisation of the beam quality in $\mathrm{CW}$ operation was limited by the onset of the thermal rollover, we continued the investigation in QCW mode. Here, the seed source and the ridge section of the tapered amplifiers remain in CW while the current into the tapered section $\left(\mathrm{I}_{\mathrm{tp}}\right)$ is pulsed at low duty cycles $(<10 \%)$ and pulse durations in the ms-range. Power measurements with the ELOD2 show that the limitations linked to the thermal rollover can be overcome allowing to reach $>10 \mathrm{~W}$ per amplifier. However concerning the use for CBC one has to consider also the power content in the central lobe which decreases significantly for higher currents ${ }^{17}$ (see Figure 7 ). We measured $>65 \%$ of the power in the central lobe for $\mathrm{I}_{\mathrm{tp}}<15$ A (Fig. 6(c)), which is still a reasonable value considering the high drive current of the tapered section. We will discuss in section 4 the challenges of $\mathrm{CBC}$ in $\mathrm{QCW}$ and present primary results.

(a)

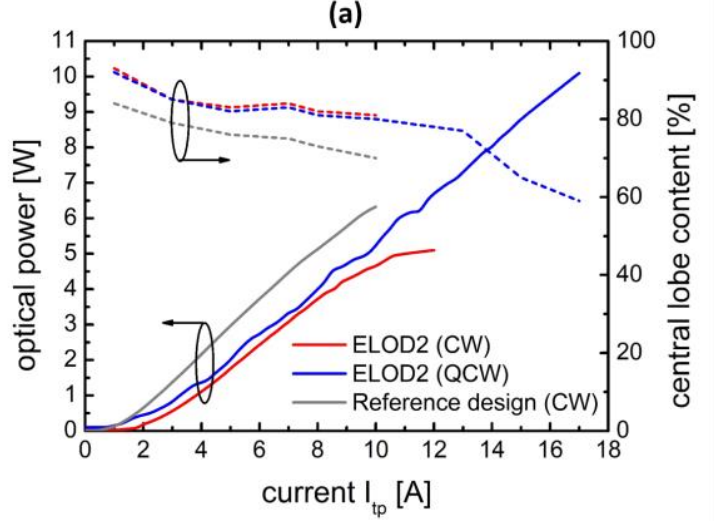

ELOD2, $Q C W, I_{T P}=8 \mathrm{~A}$

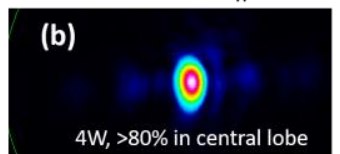

ELOD2, QCW, $\mathrm{I}_{\mathrm{TP}}=15 \mathrm{~A}$

(c)

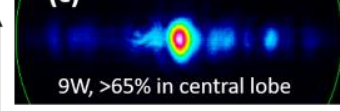

$\overrightarrow{\text { SA }}$

Figure 6: (a) Power measurements for reference (gray) and ELOD2 design in CW operation. The ELOD2 design was also measured in QCW operation (blue) with $1 \mathrm{~ms}$ on-time and a duty cycle of 10\%. (b) Beam profile at waist of an amplifier with ELOD2 design at 8 $\mathrm{A}(\mathrm{CW})$. (c) Beam profile at waist of an amplifier with ELOD2 design at $15 \mathrm{~A}(\mathrm{QCW})$. The ridge section was operated continuously for all measurements $\left(I_{R W}=350 \mathrm{~mA}\right)$. The input power was $15 \mathrm{~mW}$ for all measurements and the heatsink temperature was $20^{\circ} \mathrm{C}$. The power content in the central lobe was calculated comparing the power contained in an ideal Gaussian beam fitted to the central lobe.

Figure 7 shows a comparison of the ELOD2 design and the reference design for CBC of two amplifiers in CW mode. The CBC interferometer was identical to the experimental setup described in section 2 . The combining efficiency can indeed be improved by using the ELOD2 design and was measured to be $>85 \%$ at $10 \mathrm{~A}$, which is significantly higher than the 74 $\%$ achieved with the reference design. The combined power for the use of the ELOD2 design is $8 \mathrm{~W}$, slightly lower than for the reference design with two amplifiers. 


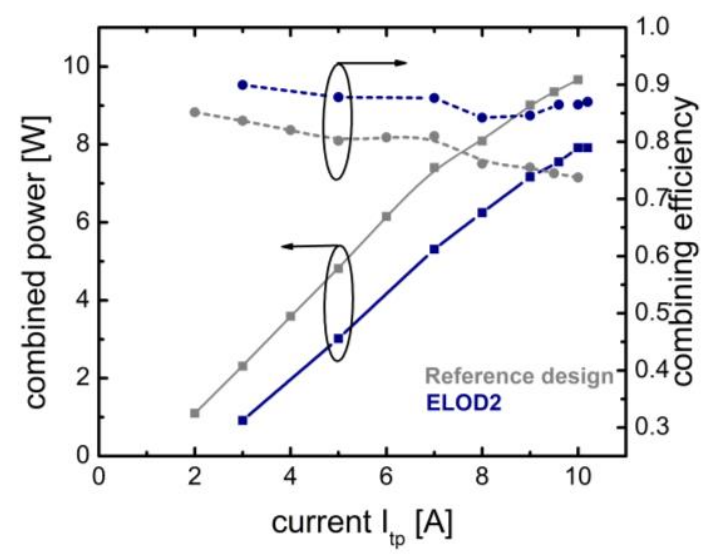

Figure 7. Power and combining efficiency for two amplifiers with the ELOD2 (blue) and with the reference design (red) in CW for different currents into the tapered section ( $\left.\mathrm{I}_{\mathrm{tp}}\right)$. The injected seed power was $15 \mathrm{~mW}$ for all amplifiers and the heatsink temperature was $20^{\circ} \mathrm{C}$.

Overall, the ELOD2 epitaxial design is a promising candidate for further studies into CBC of TPAs even if the CBC power per device is slightly lower than in our previous design. The good beam quality of the amplifiers allows to improve the combining efficiency. We are currently investigating different types of ELOD2 amplifiers to improve the power per device by an optimized device design and packaging.

\section{CBC IN QUASI CONTINOUS WAVE OPERATION}

Operation in QCW mode allows to overcome the thermal limitations in the amplifiers and to test the potential of the devices at higher powers as shown earlier in Figure 7. Additionally, CBC of tapered amplifiers operated in QCW mode can be useful for the development of modulated high power visible laser sources to address medical applications requiring millisecond and microsecond pulses ${ }^{18,19}$. The challenge of CBC in QCW is to control the turn-on dynamics in the amplifiers especially regarding the alignment and phase matching between the different beams in the CBC-interferometer.

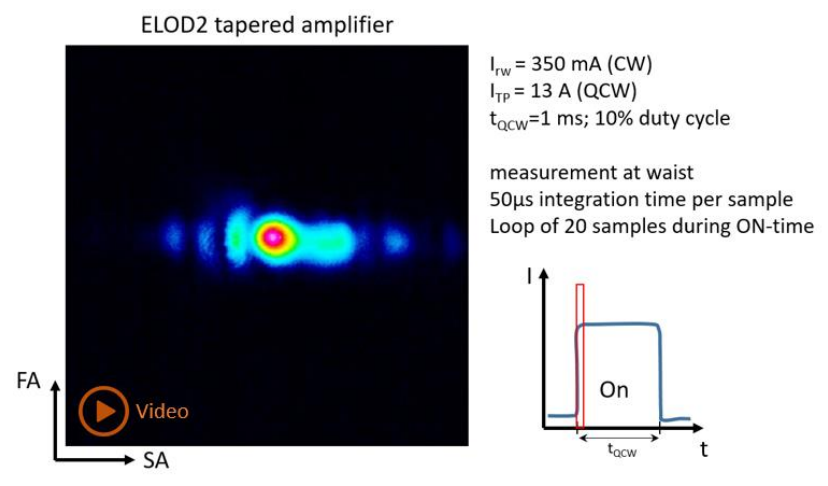

Video 1. A label of the video "spatial_dynamics_QCW.mp4". The video was assembled out of beam shape measurements triggered on the QCW pulses. Each sample was recorded with $50 \mu$ s integration time during the on-time $\tau_{\mathrm{QCW}}$ of $1 \mathrm{~ms}$. The video therefore represents the spatial dynamics of the beam at the start of each pulse. The measurement plane was chosen at the waist of the stabilized beam at the end of each pulse. Link to the video: http://dx.doi.org/doi.number.goes.here.

We analysed the spatial turn-on dynamics by triggering a fast infrared CCD camera on the QCW pulses amplified in the amplifiers. We then scanned the pulse from the start to the end of the pulse and converted the recorded samples into Video 1. The beam shape is changing quite significantly in SA at the start of the pulse. After about $250 \mu \mathrm{s}$ the beam shape is stabilized and identical to the beam shape in CW mode. The beam shape in FA is constant over the whole pulse. We interpret this effect as the thermal turn-on dynamics of the gain guided tapered section of the amplifier. The spatial dynamics of the beam shown in Video 1 are caused by a changing astigmatism as the device temperature and the current into the tapered section are changing rapidly at the start of the pulse ${ }^{20}$. We investigated different chips of the same type 
and measured very similar spatial dynamics. The power content in the central lobe is decreasing at high drive currents as shown earlier in Fig. 6. CBC of tapered amplifiers in QCW mode requires thus the precise alignment and overlap of the beams all along the pulse duration.

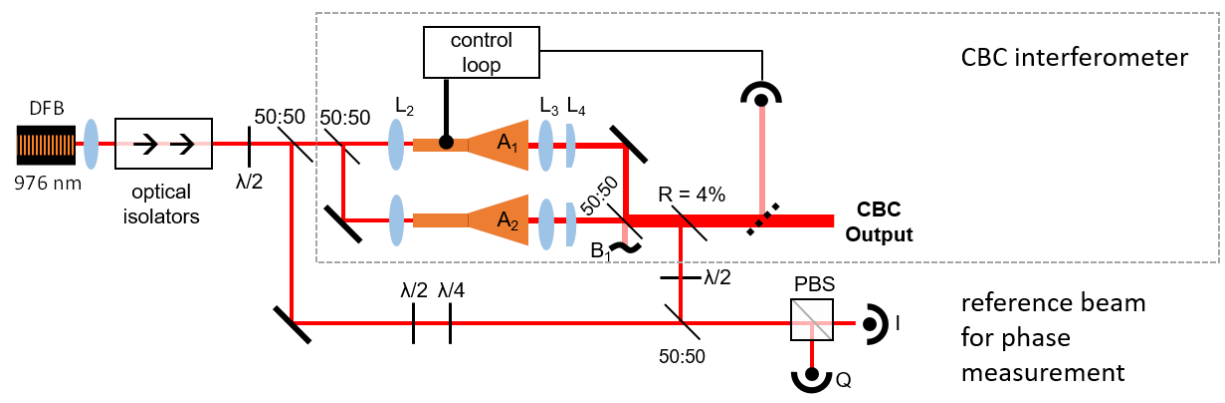

Figure 8. Experimental setup of the CBC-Interferometer and for phase measurements. PBS; Polarizing beam splitter; L1: $\mathrm{f}=8 \mathrm{~mm}$, $\mathrm{NA}=0.5 ; \mathrm{L} 2: \mathrm{f}=8 \mathrm{~mm}, \mathrm{NA}=0.5$. L3: $\mathrm{f}=2.75, \mathrm{NA}=0.55, \mathrm{~L} 4: \mathrm{f}=19 \mathrm{~mm}$. We used a triple stage faraday isolator with an optical isolation better than $-72 \mathrm{~dB}$.

The dynamics of the phase in the amplifier is important for CBC in QCW mode. We therefore implemented a reference path for phase measurements in the experimental setup shown in figure 8. We used an interferometer with two amplifiers similar to the interferometer used in Fig 1. Here, we used the third arm in the interferometer as the free space reference for phase measurements. A quarter-wave $(\lambda / 4)$ plate in the reference beam separates in-phase (I) and quadrature components (Q) with a polarising beam splitter (PBS) after the interference with a small part of the amplified beams on a non-polarising 50:50 beam combiner ${ }^{21}$. The dynamic phase shift $\varphi_{Q C W}(t)$ induced by the QCW operation of the tapered section in the amplifiers is determined using the relation $\varphi_{Q C W}(t)=-\operatorname{atan}(Q / I)$. Very high optical isolation (better than $-72 \mathrm{~dB}$ ) of the DFB seed source was mandatory during these experiments as the optical feedback of overdriven tapered amplifiers is very strong and disturbs the seed laser even at very low feedback levels ${ }^{22}$. The measured phase drift is shown for both amplifiers in Fig. 9 (a) for QCW operation of the tapered section at $13 \mathrm{~A}$. The drift of the phase within the on-time $\tau_{\mathrm{QCW}}=5 \mathrm{~ms}$ is about $30 \pi$ and is caused by a rising temperature in the amplifier during the pulses. The phase drift is however very stable from pulse to pulse and almost identical for A1 (red solid line) and A2 (dotted blue line).

We then operated the two ELOD2 amplifiers in QCW regimes in the CBC-Interferometer. As the relative differences in the phase drift in between the amplifiers are quite small $\left(\sigma_{\varphi}<\pi / 20\right)$, it is sufficient to correct a phase piston at the start of the pulse for $\mathrm{CBC}$ and there is no need to correct the phase within a QCW pulse. We used a simple hill climbing algorithm with active feedback on the ridge current of A1 (300 mA $<$ Irw $<400 \mathrm{~mA})$ to maximise the combined output power. The obtained pulse shape shown in Figure 9 (b) is stable during the pulse with a small onset of thermally induced power losses towards its end. The plateau shows small periodic fluctuations which are caused by resonance effects between the AR coated facets of the amplifier. The rise time $(\sim 250 \mu \mathrm{s})$ was limited by the current driver.
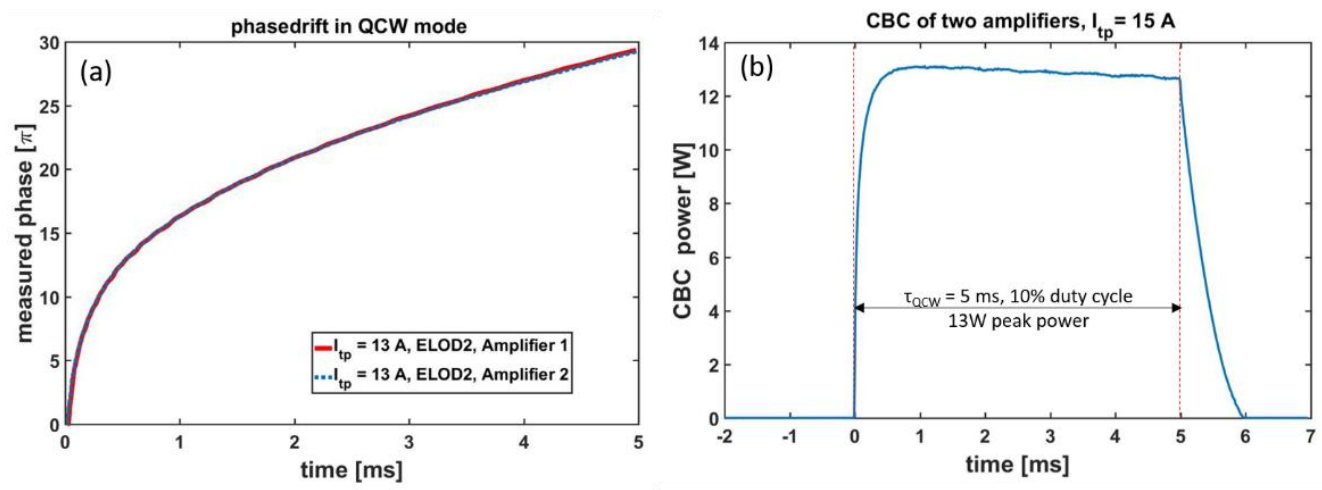

Figure 9. (a) Phase measurements for two ELOD2 amplifiers in QCW mode. (b) Photodiode measurement of the coherently combined beam from two ELOD2 amplifiers. $300 \mathrm{~mA}<\mathrm{I}_{\mathrm{rw}}<400 \mathrm{~mA}\left(\mathrm{CW}\right.$, used for phase piston control), $\mathrm{I}_{\mathrm{tp}}=13 \mathrm{~A}(\mathrm{QCW}), \tau_{\mathrm{QCW}}=5 \mathrm{~ms}, 10$ $\%$ duty cycle, heatsink $\mathrm{T}=20^{\circ} \mathrm{C}, \mathrm{P}_{\text {in }}=15 \mathrm{~mW}$ per amplifier. 
Fig. 10 shows the $\mathrm{CBC}$ power and the combining efficiency for different drive currents into the tapered section. At $15 \mathrm{~A}$ we measured $13 \mathrm{~W}$ with $>70 \%$ combining efficiency, which equals the power obtained with the three amplifiers (reference design) in CW mode (see section 2). To our knowledge, this is the first demonstration of CBC of high brightness semiconductor amplifiers in QCW mode. This experiment underlines the advantage of the use of tapered amplifiers for $\mathrm{CBC}$ architectures, as the combined power per element compares favorably to previous demonstrations of CBC of other types of semiconductor lasers and amplifiers ${ }^{23-25}$. Scaling to even higher currents is currently limited by degraded pulse stability caused by self lasing of the amplifiers. Higher power in the infrared could then be reached by multiplying the number of combined amplifiers in a larger interferometer. We are also investigating different types of ELOD2 amplifiers to gain a deeper insight into the spatial and phase dynamics at high currents.

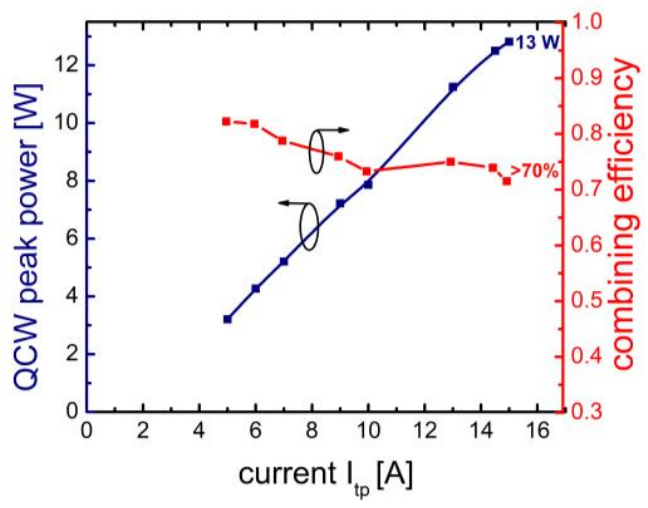

Figure 10. Power (QCW peak power) and combining efficiency at the output of the CBC interferometer for two ELOD2 tapered amplifiers. $\tau_{\mathrm{QCW}}=5 \mathrm{~ms}, 10 \%$ duty cycle, heatsink $\mathrm{T}=20^{\circ} \mathrm{C}, \mathrm{P}_{\mathrm{in}}=15 \mathrm{~mW}$ per amplifier.

\section{CONCLUSION}

Coherent beam combining is an important approach for power scaling of diode laser systems when high spatial quality and narrow linewidth are required. In the first part of the paper, we described CBC in CW mode in a simple architecture using three tapered amplifiers allowing to combine $12.9 \mathrm{~W}$ in one single beam. The beam quality was significantly increased by a beam clean up inherent to the CBC process resulting in an $86 \%$ power content in the central lobe. Those results compare favourably to previous demonstrations of $\mathrm{CBC}$ with tapered lasers ${ }^{4,26}$ regarding the power and stability of the setup. Additionally, the multi-arm MOPA configuration could further be scaled to larger number of amplifiers, should a larger NIR power be required.

This source was used for single pass nonlinear frequency conversion in a PPLN crystal. As compared to SHG of single tapered amplifiers, the improved beam quality of the $\mathrm{CBC}$ beam increases significantly the nonlinear conversion efficiency. A maximum SH power of $2.09 \mathrm{~W}$ was reached, limited by thermal dephasing of the PPLN ${ }^{10}$. Further increase of the output power in the visible spectral range would firstly require the use of different nonlinear crystals in order to deal with the high thermal load.

Furthermore, we reported on our ongoing research into the improvement of the achievable combining efficiency by advanced device design. A new amplifier design with improved beam quality (ELOD2 ${ }^{13}$ ) was characterized and we demonstrated that the achievable combining efficiency can be improved significantly. Additionally, further power scaling by $\mathrm{CBC}$ of tapered amplifiers operated in QCW mode, to limit the thermal load and overcome power limitations, was discussed and demonstrated. We achieved $13 \mathrm{~W}$ CBC peak power and $>70 \%$ combining efficiency with only two amplifiers. Further increase in the power is currently sought by increasing the number of combined amplifiers.

\section{ACKNOWLEDGMENT}

The authors are grateful to F. Moron at Laboratoire Charles Fabry for technical assistance with the active phase control in the $\mathrm{CBC}$ interferometer and to everyone involved into device development and mounting at the Ferdinand-Braun-Institut. 


\section{REFERENCES}

[1] Sumpf, B. and Paschke, K., "Spectrally stabilized high-power high-brightness DBR-tapered lasers in the VIS and NIR range," Proc. SPIE 10518, 1051817 (2018).

[2] Fan, T. Y., "Laser beam combining for high-power, high-radiance sources," IEEE Journal of Selected Topics in Quantum Electronics 11(3), 567-577 (2005).

[3] Goodno, G. D. and Rothenberg, J. E., "Engineering of Coherently Combined, High-Power Laser Systems," [Coherent Laser Beam Combining], Wiley-Blackwell, 1-44 (2013).

[4] Lucas-Leclin, G., Schimmel, G., Albrodt, P., Hanna, M. and Georges, P., "Coherent combining architectures for high-brightness laser diodes,” IEEE High Power Diode Lasers and Systems Conference (HPD), 49-50 (2017).

[5] Albrodt, P., Hanna, M., Moron, F., Decker, J., Winterfeldt, M., Blume, G., Erbert, G., Crump, P., Georges, P. and Lucas-Leclin, G., "Coherent combining of high brightness tapered lasers in master oscillator power amplifier configuration,” Proc. SPIE 10514, 105140T (2018).

[6] Boyd, R. W., [Nonlinear optics], Elsevier (2003).

[7] Müller, A., Marschall, S., Jensen, O. B., Fricke, J., Wenzel, H., Sumpf, B. and Andersen, P. E., "Diode laser based light sources for biomedical applications," Laser \& Photonics Reviews 7(5), 605-627 (2013).

[8] Jensen, O. B., Hansen, A. K., Muller, A., Sumpf, B., Unterhuber, A., Drexler, W., Petersen, P. M. and Andersen, P. E., "Power Scaling of Nonlinear Frequency Converted Tapered Diode Lasers for Biophotonics," IEEE Journal of Selected Topics in Quantum Electronics 20(2), 307-321 (2014).

[9] Fiebig, C., Blume, G., Kaspari, C., Feise, D., Fricke, J., Matalla, M., John, W., Wenzel, H., Paschke, K. and Erbert, G., "12 W high-brightness single-frequency DBR tapered diode laser," Electronics Letters 44(21), 12531255 (2008).

[10] Albrodt, P., Jamal, M. T., Hansen, A. K., Jensen, O. B., Blume, G., Paschke, K., Crump, P., Georges, P. and Lucas-Leclin, G., "Coherent combining of high brightness tapered amplifiers for efficient non-linear conversion," Optics Express 27(2), 928-937 (2019).

[11] Hansen, A. K., Tawfieq, M., Jensen, O. B., Andersen, P. E., Sumpf, B., Erbert, G. and Petersen, P. M., “Concept for power scaling second harmonic generation using a cascade of nonlinear crystals," Optics express 23(12), 15921-15934 (2015).

[12] Karamehmedović, E., Pedersen, C., Jensen, O. B. and Tidemand-Lichtenberg, P., "Nonlinear beam clean-up using resonantly enhanced sum-frequency mixing,” Appl. Phys. B 96(2), 409-413 (2009).

[13] Crump, P., Knigge, S., Maaßdorf, A., Bugge, F., Hengesbach, S., Witte, U., Hoffmann, H.-D., Köhler, B., Hubrich, R., Kissel, H., Biesenbach, J., Erbert, G. and Traenkle, G., "Low-loss smile-insensitive external frequency-stabilization of high power diode lasers enabled by vertical designs with extremely low divergence angle and high efficiency," Proc. SPIE 8605, 86050T (2013).

[14] Goodno, G. D., Shih, C.-C. and Rothenberg, J. E., "Perturbative analysis of coherent combining efficiency with mismatched lasers," Optics express 18(24), 25403-25414 (2010).

[15] Wang, X., Erbert, G., Wenzel, H., Crump, P., Eppich, B., Knigge, S., Ressel, P., Ginolas, A., Maassdorf, A. and Trankle, G., "17-W Near-Diffraction-Limited 970-nm Output From a Tapered Semiconductor Optical Amplifier," IEEE Photonics Technology Letters 25(2), 115-118 (2013).

[16] Crump, P., Decker, J., Winterfeldt, M., Fricke, J., Maals sdorf, A., Erbert, G. and Tränkle, G., "Development of high-power diode lasers with beam parameter product below $2 \mathrm{~mm}$ mrad within the BRIDLE project," Proc. SPIE 9348, 93480D (2015).

[17] Helal, M. A., Nyirenda-Kaunga, S. N., Bull, S. and Larkins, E. C., "Beam quality degradation processes in tapered lasers and DBR tapered lasers," IEEE High Power Diode Lasers and Systems Conference (HPD), 25-26 (2017).

[18] Gupta, B., Elagouz, M., McHugh, D., Chong, V. and Sivaprasad, S., "Micropulse diode laser photocoagulation for central serous chorio-retinopathy," Clinical \& Experimental Ophthalmology 37(8), 801-805 (2009).

[19] Vilera, M., Christensen, M., Hansen, A. K., Arulthasan, P., Noordegraaf, D., Buß, T., Jensen, O. B., Andersen, P. E. and Skovgaard, P. M. W., "2.7 W diffraction-limited yellow lasers by efficient frequency doubling of highbrightness tapered diode lasers," Optics Communications 435, 145-149 (2019).

[20] Wenzel, H., Sumpf, B. and Erbert, G., "High-brightness diode lasers," Comptes Rendus Physique 4(6), 649-661 (2003).

[21] Huang, R. K., Chann, B., Missaggia, L. J., Augst, S. J., Connors, M. K., Turner, G. W., Sanchez-Rubio, A., Donnelly, J. P., Hostetler, J. L. and Miester, C., "Coherent combination of slab-coupled optical waveguide lasers," Proc. SPIE 7230, 72301G (2009). 
[22] Tkach, R. and Chraplyvy, A., "Regimes of feedback effects in 1.5- $\mu \mathrm{m}$ distributed feedback lasers," Journal of Lightwave Technology 4(11), 1655-1661 (1986).

[23] Creedon, K. J., Redmond, S. M., Smith, G. M., Missaggia, L. J., Connors, M. K., Kansky, J. E., Fan, T. Y., Turner, G. W. and Sanchez-Rubio, A., "High efficiency coherent beam combining of semiconductor optical amplifiers," Optics letters 37(23), 5006-5008 (2012).

[24] Liu, B. and Braiman, Y., "Coherent addition of high power broad-area laser diodes with a compact VBG V-shaped external Talbot cavity," Optics Communications 414, 202-206 (2018).

[25] Levy, J. L. and Roh, K., “Coherent array of 900 semiconductor laser amplifiers,” Proc. SPIE 2382, 58-70 (1995).

[26] Schimmel, G., Doyen-Moldovan, I., Janicot, S., Hanna, M., Decker, J., Crump, P., Blume, G., Erbert, G., Georges, P. and Lucas-Leclin, G., "Rear-side resonator architecture for the passive coherent combining of high-brightness laser diodes," Optics letters 41(5), 950-953 (2016). 